\title{
THE DISK AROUND COKu TAURI/4: CIRCUMBINARY, NOT TRANSITIONAL ${ }^{1}$
}

\author{
M. J. IRELAND ${ }^{2}$ AND A. L. KRAUs ${ }^{3}$ \\ Received 2008 January 5; accepted 2008 March 20; published 2008 April 10
}

\begin{abstract}
$\mathrm{CoKu} \mathrm{Tau} / 4$ has been labeled as one of the very few known transition disk objects-disks around young stars that have their inner disks cleared of dust, arguably as a result of planetary formation. We report aperture-masking interferometry and adaptive optics imaging observations showing that $\mathrm{CoKu} \mathrm{Tau} / 4$ is in fact a near-equal binary star of projected separation $\sim 53$ mas $(\sim 8 \mathrm{AU})$. The spectral energy distribution of the disk is then naturally explained by the inner truncation of the disk through gravitational interactions with the binary star system. We discuss the possibility that such "unseen" binary companions could cause other circumbinary disks to be labeled as transitional.
\end{abstract}

Subject heading: stars: low-mass, brown dwarfs

\section{INTRODUCTION}

The lifetime of disks around young stars determines the timescale for giant planet formation. Most young stars have disks that are optically thick at all wavelengths or have no observable disk at all, whereas a small subset of young stars are in an intermediate state, thought to be in "transition" (Skrutskie et al. 1990). Many of these intermediate objects have optically thick "cold disks" with large excesses at $\sim 30 \mu \mathrm{m}$, but with much smaller or nonexistent excesses at near- and mid-infrared (Brown et al. 2007).

One model of this transition process is that in which the inner disk is cleared of material, leaving an inner hole and low accretion rates, followed $\sim 10^{5}$ yr later by a clearing of the outer disk. This inner hole could be caused by natural processes of grain growth and/or photoevaporation (Alexander et al. 2006) or the truncation of the disk as a result of dynamical interactions with companions (Lin \& Papaloizou 1979). Although the most exciting interpretation for the development of gaps is arguably truncation due to planetary formation, it is also possible to dynamically truncate disks due to stellar companions (e.g., Jensen \& Mathieu 1997; Beust \& Dutrey 2005; White \& Hillenbrand 2005). An example of this is CS Cha, which was announced as a transitional disk (Espaillat et al. 2007) shortly after its discovery as a circumbinary disk (Guenther et al. 2007). Some authors (e.g., Furlan et al. 2007) have considered the term "transitional" to be an observational term that means the spectral signature of a cleared inner disk rather than an evolutionary term that implicitly applies to single stars. We use the term "cold disk" for this purpose.

$\mathrm{CoKu} \mathrm{Tau} / 4$ is a weak-lined M1.5 T Tauri star $(\mathrm{H} \alpha$ equivalent width $1.8 \AA$; Cohen \& Kuhi 1979) in the Taurus star-forming region (1-2 Myr, $\sim 145 \mathrm{pc}$ ). $\mathrm{CoKu} \mathrm{Tau} / 4$ was discovered to have a large $\sim 20-30 \mu \mathrm{m}$ excess and no excess at wavelengths $<8 \mu \mathrm{m}$, by Spitzer Space Telescope Infrared Spectrograph observations (Forrest et al. 2004). The disk has been modeled as having an inner hole of radius $\sim 10$ AU (D'Alessio et al. 2005), and there

\footnotetext{
${ }^{1}$ Data presented herein were obtained at the W. M. Keck Observatory, which is operated as a scientific partnership among the California Institute of Technology, the University of California, and the National Aeronautics and Space Administration. The Observatory was made possible by the generous financial support of the W. M. Keck Foundation.

${ }^{2}$ Division of Geological and Planetary Sciences, California Institute of Technology, Pasadena, CA 91125; mireland@gps.caltech.edu.

${ }^{3}$ Division of Physics, Mathematics and Astronomy, California Institute of Technology, Pasadena, CA 91125.
}

have been suggestions that this hole is due to a giant planet (Quillen et al. 2004).

In this Letter, we describe near-infrared aperture-masking interferometry and imaging observations that demonstrate that $\mathrm{CoKu} \mathrm{Tau} / 4$ is a near-equal mass binary star system. We show that the predicted inner hole size from dynamical models is comparable to, but larger than, that predicted from radiative transfer models. Finally, we discuss whether other so-called transitional disks could be circumbinary disks. We conclude that, for candidates in Taurus, much of the mass ratio-separation space for stellar companions can by ruled out by existing observations but that definitively ruling out the possibility of binarity is generally difficult for individual so-called transitional disks.

\section{OBSERVATIONS}

$\mathrm{CoKu} \mathrm{Tau} / 4$ was observed with the NIRC2 camera behind adaptive optics (AO) at the Keck II telescope on 2007 November 23 as part of an ongoing aperture-masking survey of nearby young star-forming associations. Aperture-masking interferometry (e.g., Tuthill et al. 2000) is a well-established technique for achieving the full diffraction limit of a single telescope, recently applied to observations behind AO systems (e.g., Lloyd et al. 2006; Kraus et al. 2008). A nine-hole mask was placed in a filter wheel near a pupil plane in the NIRC2 camera, enabling interference fringes on 36 baselines to be simultaneously recorded on the camera's imaging array. The observations of $\mathrm{CoKu} \mathrm{Tau} / 4$ consisted of two image sets taken through a $K^{\prime}$-filter, each with eight $20 \mathrm{~s}$ exposures, calibrated by two interleaved image sets of CX Tau. The air mass of $\mathrm{CoKu} \mathrm{Tau} / 4$ observations varied from 1.28 to 1.38 , whereas the air mass of CX Tau ( $6^{\circ}$ from $\left.\mathrm{CoKu} \mathrm{Tau} / 4\right)$ varied from 1.32 to 1.45 over the 45 minutes it took to complete the observations. From the Fourier transforms of the target and calibrator's images, squared visibilities and closure phases were extracted. The squared visibilities of $\mathrm{CoKu} \mathrm{Tau} / 4$ were calibrated by dividing by the squared visibilities of the calibrator, and the closure phases were calibrated by subtracting the closure phases of the calibrator (which are nonzero due to instrumental effects). These observations were made in poor seeing conditions (uncorrected seeing $1^{\prime \prime}-2$ " in the $K$ band), so our observations only achieved typical sensitivities of $\sim 50: 1$ at the diffraction limit, less than the 200 : 1 we achieved for Upper Sco (Kraus et al. 2008). These high sensitivities are primarily due to the nonredundant nature of the aperture mask, which means that calibration is independent of wave-front structure on scales larger than a single subaperture $(\sim 1 \mathrm{~m})$. 


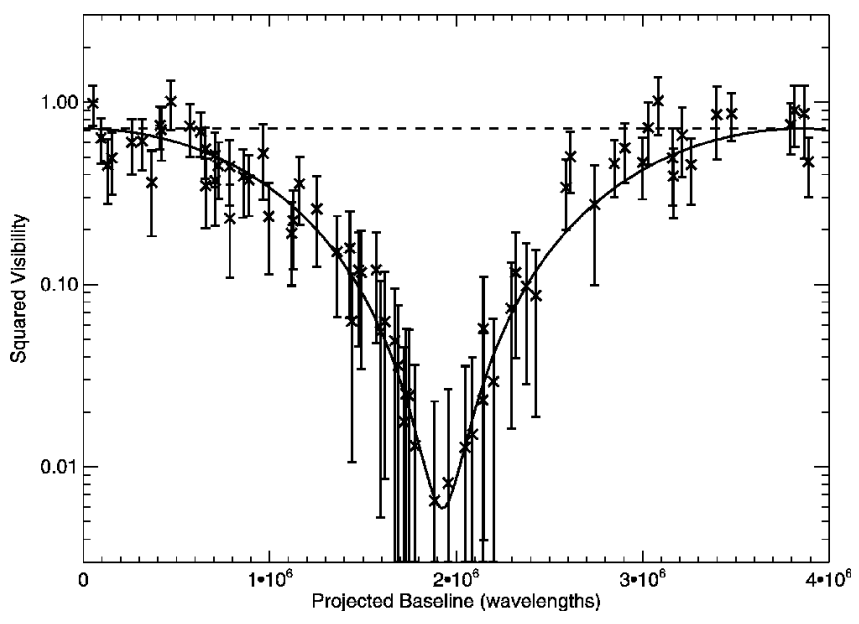

FIG. 1.-Calibrated squared visibilities for CoKu Tau 4, plotted against a baseline projected along a position angle of $306^{\circ}$. A binary model with contrast 1.25 and separation 54 mas is overplotted. The dashed line shows the visibility expected for a single star.

We found that only a binary model provided a good fit to the aperture-masking data, with a near-equal binary well within our detection limits. The calibrated squared visibilities and closure phases are shown in Figures 1 and 2, demonstrating that a binary solution provides an excellent fit to the data. The binary solution, based on the aperture-masking data, is given in Table 1.

Preliminary analysis of the masking data indicated that the binary companion's separation and flux ratio would allow it to be resolved with direct imaging, so we obtained regular AO images on the following night under slightly better observing conditions (uncorrected seeing $\sim 1^{\prime \prime}$ in the $K$ band) at an air mass of 1.06. These observations were obtained with NIRC2 in the narrow camera mode $\left(\sim 10\right.$ mas pixel $\left.^{-1}\right)$, and since the target is very bright in the near-infrared, we used the narrowband filters $J_{\text {cont }}, H_{\text {cont }}, K_{\text {cont }}$, and $\mathrm{Br} \gamma$. Total exposure times were $40 \mathrm{~s}$ in all filters except for $J_{\text {cont }}$, in which twice as many images were taken due to the very low Strehl, for a total exposure time of $80 \mathrm{~s}$. We show the images in Figure 3. Despite the low Strehl ratio and the elongation of the speckle halo due to a dominant wind direction, the binary is clearly resolved in all images.

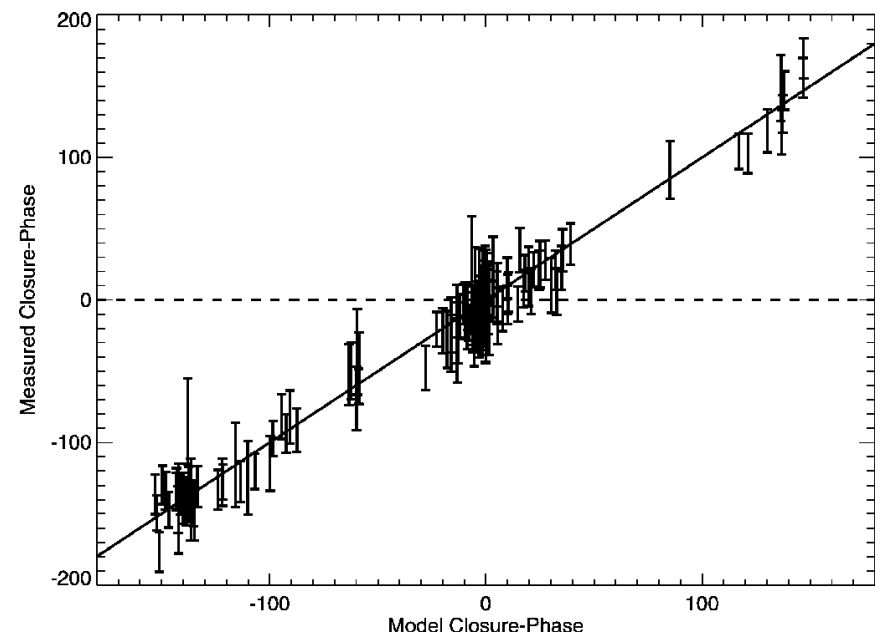

FIG. 2.-Measured closure phases for CoKu Tau 4 as a function of modeled closure phases, assuming that CoKu Tau 4 is a $1.25: 1$ binary with separation 54 mas at a position angle of $306^{\circ}$. The dashed line shows the zero closure phase expected for a single star.
TABLE 1

Direct ImAging OBSERvations

\begin{tabular}{|c|c|c|c|c|}
\hline Filter & $\begin{array}{c}\text { Wavelength } \\
\text { Range } \\
(\mu \mathrm{m})\end{array}$ & $\begin{array}{c}\rho \\
\text { (mas) }\end{array}$ & $\begin{array}{l}\text { Position } \\
\text { Angle } \\
\text { (deg) }\end{array}$ & $\underset{(\mathrm{mag})}{\Delta}$ \\
\hline \multicolumn{5}{|c|}{ Aperture Masking } \\
\hline$K^{\prime} \ldots$ & $1.948-2.299$ & $54.1 \pm 0.3$ & $306.7 \pm 0.4$ & $0.23 \pm 0.01$ \\
\hline \multicolumn{5}{|c|}{ PSF Fitting } \\
\hline$H_{\text {cont }}$ & $1.569-1.592$ & $53.6 \pm 0.7$ & $306.3 \pm 0.3$ & $0.24 \pm 0.04$ \\
\hline $\mathrm{Br} \gamma \ldots$ & $2.152-2.185$ & $53.3 \pm 0.2$ & $307.7 \pm 0.7$ & $0.25 \pm 0.01$ \\
\hline$K_{\text {cont }} \cdots \cdots$ & $2.256-2.285$ & $53.6 \pm 0.2$ & $307.1 \pm 0.2$ & $0.21 \pm 0.01$ \\
\hline \multicolumn{5}{|c|}{ Bispectrum Analysis } \\
\hline & $1.203-1.223$ & $51.7 \pm 0.9$ & $305.8 \pm 0.6$ & $0.23 \pm 0.06$ \\
\hline$H_{\text {cont }} \ldots \ldots$ & $1.569-1.592$ & $53.3 \pm 0.2$ & $306.9 \pm 0.2$ & $0.21 \pm 0.02$ \\
\hline $\operatorname{Br} \gamma \ldots \ldots$ & $2.152-2.185$ & $53.9 \pm 0.2$ & $306.4 \pm 0.2$ & $0.19 \pm 0.01$ \\
\hline$K_{\text {cont }} \cdots \cdots$ & $2.256-2.285$ & $53.6 \pm 0.2$ & $306.0 \pm 0.2$ & $0.20 \pm 0.01$ \\
\hline
\end{tabular}

We extracted photometry and astrometry from the direct AO imaging of these sources using the point-spread function (PSF) reconstruction technique described in Kraus \& Hillenbrand (2007b); this technique iteratively fits a template PSF to the primary and then subtracts the secondary to fit an improved estimate of the primary. This routine was implemented using the ALLSTAR routine in the IRAF package DAOPHOT (Stetson 1987), and on the basis of previous experience with NIRC2 imaging, we chose to model the PSF using a Gaussian core with Lorentzian wings. This method is illustrated in Figure 3. In addition to this method, we used a recursive-phase algorithm (e.g., Lohmann et al. 1983) to reconstruct the Fourier phase from the bispectrum phase and then fitted a binary model to the reconstructed phase of the image. In Table 1, we summarize the relative astrometry and photometry that we measured from our direct imaging observations. The consistency in separation and position angle at the $1 \%$ level in the $H$ and $K$ bands and at the $5 \%$ level in the $J$ band, despite very different PSFs and diffraction effects, demonstrates the reliability of our results. However, the small statistical errors reported in Table 1 are not entirely consistent internally, requiring additional systematic errors to be added. Therefore, we assign a weighted mean
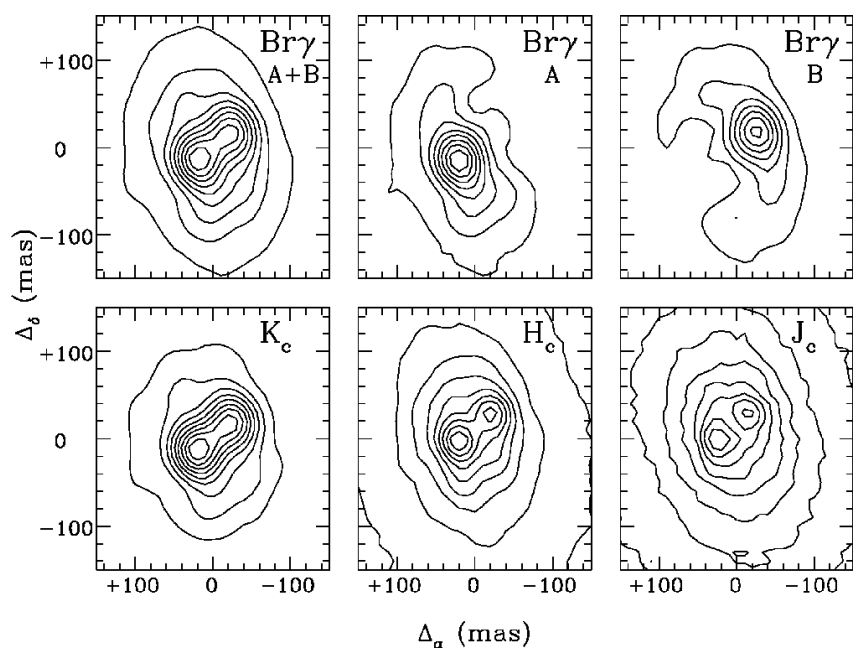

FIG. 3.-Top panels: Contour plots of the original AO image, the A-component only (with the best-fit B-solution subtracted), and the B-component only (with the best-fit A-solution subtracted) in the Br $\gamma$-filter, demonstrating the PSF-fitting technique. Bottom panels: AO images in the $K_{\text {cont }}, H_{\text {cont }}{ }^{-}$, and $J_{\text {cont }}$-filters, clearly showing the binary despite the low Strehl. The contours are drawn at $10 \%-90 \%$ in intervals of $10 \%$. 
solution of separation $53.6 \pm 0.5$ mas and a position angle of $306.4^{\circ} \pm 0.6^{\circ}$. The contrast ratio in $J, H$, and $K$ is consistent with there being no significant difference in reddening between the components.

\section{SYSTEM PROPERTIES}

\subsection{Stellar and Binary Properties}

Both components have near-infrared colors that are consistent with other young Taurus members of spectral type $\sim \mathrm{M} 1-$ M2, suggesting that they have similar temperatures and that neither has a significant $K$-band excess due to a hot inner disk. In order to model the stars and the disk, it is important to know the bolometric luminosity of the system and, hence, the reddening. To estimate the reddening, we used the following data: intrinsic colors reported in Bessell \& Brett (1988) and Bessell (1990) interpolating between giants $(\log g \sim 1)$ and dwarfs $(\log g \sim 5)$ at an assumed $\log g$ of $3.7, J H K$ photometry from 2MASS, $V$-band photometry averaged between Cohen \& Kuhi (1979) and Hanson et al. (2004) with an assumed 0.1 mag overall uncertainty, and an M1.5 spectral classification from Cohen \& Kuhi (1979) with an assumed 1 subclass uncertainty. We arrive at $E_{V-K}=2.3 \pm 0.3$ and $E_{J-K}=0.58 \pm 0.04$. The $E_{V-K} / E_{J-K}$ ratio is $4.0 \pm 0.6$, which is significantly lower than the value of 5.6 typical of Taurus (Whittet et al. 2001), suggesting a reddening law more typical of a dense core. Based on $E_{V-K}$, the extinction $A_{V}$ is $2.5 \pm 0.3$ using the relationship $A_{V} \approx 1.1 E_{V-K}$ from Whittet et al. (2001), but $A_{V}=3$ as used by D'Alessio et al. (2005) is certainly consistent, given the uncertainties in the reddening law.

Absolute stellar properties like masses can be difficult to estimate because pre-main-sequence evolutionary models are not well calibrated, but based on the prescription described in Kraus \& Hillenbrand (2007a), both components are likely to have masses of $\sim 0.5-0.6 M_{\odot}$. Using a $145 \mathrm{pc}$ distance to Taurus, the system has an absolute $K$ magnitude of 2.55 , which implies absolute $K$ magnitudes of 3.2 and 3.4 for the components. According to the models of Baraffe et al. (1998), assuming an effective temperature of $\sim 3600 \mathrm{~K}$ applicable to M1.5 stars (Luhman 1999), the stars are 4 Myr old and are of masses $\sim 0.52$ and $\sim 0.62 M_{\odot}$. If the effective temperature is closer to $3500 \mathrm{~K}$, the stars would be of slightly lower mass and <3 Myr old, consistent with the canonical age of Taurus. The observational scatter in the Taurus H-R diagram (e.g., Luhman 2004) is consistent with $\mathrm{CoKu} \mathrm{Tau} / 4$ being either single, as assumed by previous authors, or an equal-brightness double. The mass ratio, however, should be more robust than the masses or age (as it is only weakly dependent on reddening), so we can assert with greater confidence that $M_{B} / M_{A}=0.85 \pm 0.05$.

The minimum possible semimajor axis $(a)$ for the orbit is the apparent separation divided by $(1+e)$, which is $7.8 /(1+$ e) AU based on an assumed distance of $145 \mathrm{pc}$ for Taurus, giving an orbital period of $>10$ years. Based on considerations of dynamical disk truncation in $\S 3.2$, it is likely that the system was observed near maximum elongation.

\subsection{The Circumbinary Disk}

We expect the inner radius of the $\mathrm{CoKu}$ Tau/4 disk to be set by tidal truncation. To simplify our discussion, we will assume that the disk is roughly coplanar with the binary and (as described in $\S 3.1$ ) that the binary components have similar masses. In this geometry, Artymowicz \& Lubow (1994) calculate that the $3: 1$ orbital resonance at $R_{D} \sim 2.08 a$ is opened for all but improbably small eccentricities $(e \gtrsim 0.03)$. Beust $\&$ Dutrey (2005) predict a gap size of $>2.6 a$ for eccentricities $>0.1$ for GG Tau, which has a similar mass ratio to CoKu Tau/ 4 (and find this not to be large enough for that system). For very large eccentricities ( $e \gtrsim 0.6)$, larger resonances open, and predicted gap sizes from Artymowicz \& Lubow (1994) are in excess of $R_{D} \sim 3 a$ but are viscosity-dependent. The closer nearequal spectroscopic binary HD 98800B (Akeson et al. 2007) is an example of a system with high eccentricity that should have opened large resonances in the disk, but the radiative transfer modeling has too many uncertainties to clearly say which resonance has opened.

Based on the minimum semimajor axis of $7.8 /(1+e) \mathrm{AU}$ ( $\$ 3.1$ ), we arrive at a minimum disk truncation radius of $\sim 16 \mathrm{AU}$ if $\mathrm{CoKu}$ Tau/4 is a low $(e \sim 0.1)$ eccentricity binary or $\sim 13 \mathrm{AU}$ if it is a high ( $e \geqslant 0.6$ ) eccentricity binary. These numbers are discrepant from the 9-12 AU truncation radius predicted by the detailed spectral modeling of D'Alessio et al. (2005), who examined radiative transfer models consisting of a geometrically thin wall and a range of dust types. We will examine several ways to resolve this discrepancy in turn: the differing radiation pattern of a binary versus a single star, errors in the stellar luminosity, and the possibility of a substantially different radiative transfer model.

A binary star's radiation pattern is different from a single star's. In particular, on a circular ring of radius $R_{d}$ centered on the binary center of mass, the flux is greatest at points on this ring aligned with the axis of the binary. For an 8 AU binary (each component $4 \mathrm{AU}$ from the center of mass) and a $14 \mathrm{AU}$ ring, this geometry means that the flux from the binary is between 0.92 and 1.28 times the flux from a single star with the same total luminosity. We will assume that the $\sim 145 \mathrm{~K}$ modeled by D'Alessio et al. (2005) represents the maximum temperature seen in the ring, and this temperature determines $L_{S} / R_{D}^{2}$. Here $L_{S}$ is the total stellar luminosity, and $R_{D}$ is the dust radius from the center of mass. Therefore, a $28 \%$ increase in flux $F$ could represent an $\sim 13 \%$ increase in the ring radius from radiative transfer modeling. The temperature contrast around the ring for optically thin dust with opacity $\kappa_{\lambda} \propto \lambda^{-1}$ goes as $F^{1 / 5}$, meaning that the temperature around the ring ranges from $\sim 136$ to $145 \mathrm{~K}$. Gray dust would vary from $\sim 133$ to $145 \mathrm{~K}$. Although this contrast is only moderate, it may present an additional challenge to detailed spectral fitting, as the models of D'Alessio et al. (2005) required emission from a single temperature only.

One way to reconcile the gap radius from radiative transfer modeling with that from dynamical considerations would be if the luminosity of $\mathrm{CoKu} \mathrm{Tau} / 4$ is significantly underestimated. If we keep the dust temperature, and therefore $L_{S} / R_{D}^{2}$, constant, increasing $L_{S}$ increases the model $R_{D}$. An uncertainty in distance due to the depth of Taurus (e.g., Torres et al. 2007) cannot reconcile this discrepancy, as both $R_{D}$ determined from radiative transfer modeling and the minimum $R_{D}$ determined by dynamical considerations increase linearly with the distance estimate. However, an uncertainty in the reddening of $\mathrm{CoKu} \mathrm{Tau} / 4$ translates to a luminosity uncertainty. The probable reddening calculated in $\S 3.1$ is lower than that used by D'Alessio et al. (2005), but uncertainties are considerable, and it may be possible that a larger bolometric reddening could increase the gap radius derived from radiative transfer modeling.

In order to independently estimate the dust radius from radiative transfer modeling and appreciate possible further complexities, we will examine a very simple model. This model contains small grains of amorphous olivine (i.e., the limit in 
which the grain radius is much smaller than the blackbody emission peak), with optical constants from Dorschner et al. (1995). We assign a dust temperature $T_{D}=145 \mathrm{~K}$ and a stellar luminosity of $0.61 L_{\odot}$, as in D'Alessio et al. (2005), and a stellar temperature of an M1.5 star of $T_{S}=3600 \mathrm{~K}$ (Luhman 1999). The stellar radius for each of the two stars is $3.7 R_{\odot}$, equivalent to a single star of radius $5.2 R_{\odot}$. We assume a blackbody spectrum for the star. ${ }^{4}$ The dust is assumed optically thin (which could represent the outer layers of an optically thick disk), so that the dust radius is then given by

$$
R_{D}=R_{S} \sqrt{\frac{\int \kappa_{\lambda} B_{\lambda}\left(T_{S}\right) d \lambda}{2 \int \kappa_{\lambda} B_{\lambda}\left(T_{D}\right) d \lambda}} .
$$

In this simple model, the dust radius is $R_{D}=15 \mathrm{AU}$, roughly consistent with disk truncation radii from dynamical considerations. With the many possible parameters to tweak in a more complete model, and the possibility of the optically thin dust being additionally heated by surrounding dust, increasing $T_{D}$ and driving the model radius outward, it appears likely that a detailed radiative transfer model could indeed be made to match the dynamical truncation radius. The edge in the spectrum of disk emission from $\mathrm{CoKu} \mathrm{Tau} / 4$ at $\sim 9 \mu \mathrm{m}$ coincides with a sharp rise in silicate emissivity, meaning that the interplay between temperature, extinction, geometry, and dust composition is all the more complex for this source. We hope that this preliminary discussion will motivate other authors to examine possible models in more detail.

\section{DISCUSSION AND CONCLUSIONS}

The circumbinary nature of the $\mathrm{CoKu}$ Tau/4 disk begs the following question: are other so-called transition disks also likely circumbinary in nature? The other well-known cold disks in Taurus (DM Tau, LkCa 15, UX Tau, and GM Aur) are also generally assumed to be single stars. Although all these systems differ from one another, $\mathrm{CoKu} \mathrm{Tau} / 4$ is perhaps the most unique because of its very low accretion rate. So far, with our aperture-

\footnotetext{
${ }^{4}$ Assuming instead the NextGen model spectrum from Hauschildt et al. (1999) at $3600 \mathrm{~K}$ and $\log g=4.0$, we find that $R_{D}$ is reduced by $2 \mathrm{AU}$, but it has far too much $\mathrm{TiO}$ absorption when compared to an M1.5 spectrum
}

masking observations of these systems (M. J. Ireland et al., in preparation), we can eliminate stellar (mass ratio $q>0.1$ ) companions over a 20-160 mas projected separation range, but we can say very little about the possibility of closer companions. Accurate $\left(\sigma \lesssim 1 \mathrm{~km} \mathrm{~s}^{-1}\right)$ radial velocity monitoring of these systems is required to determine if any of them harbor a close ( $\lesssim 4 \mathrm{AU}$ ) companion. Radial velocity monitoring is most applicable to DM Tau, where the cleared inner hole is expected to have only a 3 AU radius (Calvet et al. 2005). Simon et al. (2000) used resolved CO 2-1 observations of disks around several $\mathrm{T}$ Tauri stars to measure their masses directly. These measurements would have resulted in the total mass of the stellar primaries and any unresolved companions. The total masses of $0.84 \pm 0.05$, $0.97 \pm 0.03$, and $0.55 \pm 0.03$ for GM Aur, LkCa 15, and DM Tau, respectively, provide convincing evidence that these systems do not harbor companions with mass ratios $q>0.3$.

In summary, CoKu Tau/4 is a binary star system with a current projected separation of $\sim 7.8 \mathrm{AU}$ and a mass ratio near unity. The disk surrounding $\mathrm{CoKu} \mathrm{Tau} / 4$ is therefore a circumbinary disk, with the disk inner edge set by dynamical truncation. The dynamical disk truncation radius of $\gtrsim 13-16$ AU is inconsistent with the disk radius derived from previous spectral modeling, but it is likely that a different choice of reddening law and correction, dust composition, and geometry will resolve this small discrepancy. We suggest that systematic radial velocity monitoring and aperture-masking surveys are required to determine if other so-called transition disks are in fact multiple systems.

We gratefully acknowledge helpful discussions with Lynne Hillenbrand, Klaus Pontoppidan, Geoffrey Blake, Gregory Herczeg, and Colette Salyk and acknowledge the assistance of Thierry Forveille in pointing out an oversight in an early version of the manuscript. M. J. I. would like to acknowledge Michelson Fellowship support from the Michelson Science Center and the NASA Navigator Program. A. L. K. is supported by a NASA/Origins grant to L. Hillenbrand. The authors wish to recognize and acknowledge the very significant cultural role and reverence that the summit of Mauna Kea has always had within the indigenous Hawaiian community. We are most fortunate to have the opportunity to conduct observations from this mountain.

Facility: Keck:II(NIRC2)

\section{REFERENCES}

Akeson, R. L., Rice, W. K. M., Boden, A. F., Sargent, A. I., Carpenter, J. M., \& Bryden, G. 2007, ApJ, 670, 1240

Alexander, R. D., Clarke, C. J., \& Pringle, J. E. 2006, MNRAS, 369, 229

Artymowicz, P., \& Lubow, S. H. 1994, ApJ, 421, 651

Baraffe, I., Chabrier, G., Allard, F., \& Hauschildt, P. H. 1998, A\&A, 337, 403

Bessell, M. S. 1990, PASP, 102, 1181

Bessell, M. S., \& Brett, J. M. 1988, PASP, 100, 1134

Beust, H., \& Dutrey, A. 2005, A\&A, 439, 585

Brown, J. M., et al., 2007, ApJ, 664, L107

Calvet, N., et al. 2005, ApJ, 630, L185

Cohen, M., \& Kuhi, L. V. 1979, ApJS, 41, 743

D'Alessio, P., et al. 2005, ApJ, 621, 461

Dorschner, J., Begemann, B., Henning, T., Jaeger, C., \& Mutschke, H. 1995, A\&A, 300, 503

Espaillat, C., et al. 2007, ApJ, 664, L111

Forrest, W. J., et al. 2004, ApJS, 154, 443

Furlan, E., et al. 2007, ApJ, 664, 1176

Guenther, E. W., Esposito, M., Mundt, R., Covino, E., Alcalá, J. M., Cusano, F., \& Stecklum, B. 2007, A\&A, 467, 1147

Hanson, R. B., Klemola, A. R., Jones, B. F., \& Monet, D. G. 2004, AJ, 128, 1430

Hauschildt, P. H., Allard, F., \& Baron, E. 1999, ApJ, 512, 377

Jensen, E. L. N., \& Mathieu, R. D. 1997, AJ, 114, 301
Kraus, A. L., \& Hillenbrand, L. A. 2007a, ApJ, 662, 413 2007b, ApJ, 664, 1167

Kraus, A. L., Ireland, M. J., Martinache, F., \& Lloyd, J. P. 2008, ApJ, submitted Lin, D. N. C., \& Papaloizou, J. 1979, MNRAS, 186, 799

Lloyd, J. P., Martinache, F., Ireland, M. J., Monnier, J. D., Pravdo, S. H., Shaklan, S. B., \& Tuthill, P. G. 2006, ApJ, 650, L131

Lohmann, A. W., Weigelt, G., \& Wirnitzer, B. 1983, Appl. Opt., 22, 4028

Luhman, K. L. 1999, ApJ, 525, 466 . 2004, ApJ, 617, 1216

Quillen, A. C., Blackman, E. G., Frank, A., \& Varnière, P. 2004, ApJ, 612, L137

Simon, M., Dutrey, A., \& Guilloteau, S. 2000, ApJ, 545, 1034

Skrutskie, M. F., Dutkevitch, D., Strom, S. E., Edwards, S., Strom, K. M., \& Shure, M. A. 1990, AJ, 99, 1187

Stetson, P. 1987, PASP, 99, 191

Torres, R. M., Loinard, L., Mioduszewski, A. J., \& Rodríguez, L. F. 2007, ApJ, 671, 1813

Tuthill, P. G., Monnier, J. D., Danchi, W. C., Wishnow, E. H., \& Haniff, C. A. 2000, PASP, 112,555

White, R. J., \& Hillenbrand, L. A., 2005, ApJ, 621, L65

Whittet, D. C. B., Gerakines, P. A., Hough, J. H., \& Shenoy, S. S. 2001, ApJ, 547,872 